\title{
The triple near-Earth asteroid (153591) 2001 SN263: an ultra-blue, primitive target for the Aster space mission $\star$
}

\author{
D. Perna ${ }^{1}$, A. Alvarez-Candal ${ }^{2}$, S. Fornasier ${ }^{1,3}$, Z. Kaňuchová ${ }^{1,4}$, S. M. Giuliatti Winter ${ }^{5}$, \\ E. Vieira Neto ${ }^{5}$, and O. C. Winter ${ }^{5}$
}

\author{
${ }^{1}$ LESIA - Observatoire de Paris, CNRS, UPMC Univ. Paris 06, Univ. Paris-Diderot, 5 place J. Janssen, 92195 Meudon, France \\ e-mail: davide.perna@obspm.fr \\ 2 Observatório Nacional, rua General José Cristino 77, 20921-400 Rio de Janeiro, Brazil \\ 3 Université Paris Diderot - Paris 7, 4 rue Elsa Morante, 75013 Paris, France \\ 4 Astronomical Institute of the Slovak Academy of Sciences, 05960 Tatranská Lomnica, Slovak Republic \\ 5 Universidade Estadual Paulista, Grupo de Dinâmica Orbital \& Planetologia, CEP 12516-410, SP Guaratinguetà, Brazil
}

Received 21 June 2014 / Accepted 17 July 2014

\section{ABSTRACT}

\begin{abstract}
Context. The Brazilian Aster project plans a space mission to rendezvous and characterize (153591) 2001 SN263, one of the only two known triple near-Earth asteroids (NEAs). Improving the knowledge of its physical properties is necessary to optimize the mission planning and science return.

Aims. We study the surface composition and physical nature of 2001 SN263 by analyzing and comparing its reflectance spectra with laboratory spectra of minerals and meteorites.

Methods. We performed spectroscopic observations of 2001 SN263 using the UV-to-NIR X-Shooter spectrograph at the ESO Very Large Telescope (VLT). Complementary photometric observations of the target were acquired with the FORS2 instrument.

Results. We find B-type, featureless convex spectra (Themis- or Polana-like). 2001 SN263 presents the bluest visible spectrum ever observed for small bodies in the solar system, even bluer than NEAs Phaethon and Bennu. The spectra suggest that the surface composition is organic- and magnetite-rich, similar to that of heated CI carbonaceous chondrites. Phyllosilicates may be abundant as well. We find hints of a coarse-grained surface and composition variety within the triple system.

Conclusions. Both the large grain size and surface variability might be connected to the formation of the triple system. The Aster mission will have the intriguing possibility of checking current models of asteroid binary formation.
\end{abstract}

Key words. minor planets, asteroids: individual: (153591) 2001 SN263 - techniques: spectroscopic - techniques: photometric

\section{Introduction}

The near-Earth asteroid (NEA) (153591) 2001 SN263 has a semimajor axis of $1.99 \mathrm{AU}$, an eccentricity of 0.48 , and an inclination of $6.7^{\circ}$. It has an absolute magnitude $H=16.9$ and an Earth minimum orbit intersection distance (MOID) of $0.05061 \mathrm{AU}$, marginally beyond that of potentially hazardous asteroids (defined to have MOID $<0.05$ AU and $H<22$ ). It is an easily accessible target for a space mission, with a velocity increment required for a spacecraft starting from low-Earth orbit to rendezvous with the asteroid (Shoemaker \& Helin 1978) of $\Delta V=5.96 \mathrm{~km} \mathrm{~s}^{-1}$, which is lower than that needed to reach the Moon and Mars.

2001 SN263 is one of the only two established triple systems in the NEA population. Based on radar and light-curve data, Becker et al. (2014) found that the primary is spheroidal with an equivalent diameter of $2.50 \pm 0.30 \mathrm{~km}$ and displays an equatorial bulge. Its sidereal rotation period is $3.4256 \pm 0.0002 \mathrm{~h}$. The larger satellite is elongated and has an equivalent diameter of $0.77 \pm 0.12 \mathrm{~km}$. Its sidereal rotation period is $13.439 \mathrm{~h}$, and it has an orbital period of approximately six days. The smaller satellite is in a closer orbit around the primary, has an equivalent diameter of $0.43 \pm 0.14 \mathrm{~km}$, and is probably tidally locked to the primary. The derived densities of the primary, the larger satellite, and the

* Based on observations carried out at the European Southern Observatory (ESO), Chile (Programme 287.C-5026). smaller satellite are $1.13 \pm 0.15 \mathrm{~g} / \mathrm{cm}^{3}, 1.01 \pm 0.41 \mathrm{~g} / \mathrm{cm}^{3}$, and $2.27 \pm 1.27 \mathrm{~g} / \mathrm{cm}^{3}$, respectively. The measured radar echo's circular polarization ratio is $0.16 \pm 0.01$ (Benner et al. 2008), suggesting a low surface roughness; Betzler et al. (2008) detected mutual events (eclipses and transits) in the light curves. The low density and circular polarization ratio, the geometric visible albedo $p_{V}=0.048 \pm 0.015$ measured by Delbo et al. (2011) from thermal infrared observations, and the available near-infrared (NIR) reflectance spectra that show an overall blue or flat slope (Reddy et al. 2008; Ostrowski et al. 2011), all point toward a primitive, carbonaceous nature for 2001 SN263.

The remarkable characteristics of 2001 SN263, including the relatively large size and spacing of the components, which make the system safe to explore (Araujo et al. 2012), have drawn the attention of planetary scientists and space agencies: formerly the target of the Amor mission (not selected for the NASA Discovery program 2010; Jones et al. 2011), this triple system is currently the primary target of the Brazilian Aster project, which is planned to reach, then orbit and study the system for about 10 months. Different possible launch dates have been identified in the 2014-2020 time frame (Sukhanov et al. 2010).

Before the present study, no ultraviolet (UV) and visible spectra of 2001 SN263 had been acquired, while a reliable compositional analysis of its surface - very important for optimizing the mission procedures of Aster - would require spectra on a wide wavelength range acquired in the same 
Table 1. Observational circumstances and measured slopes for the acquired spectra (see text for details).

\begin{tabular}{|c|c|c|c|c|c|c|c|c|}
\hline Spectrum & Date & $\mathrm{UT}_{\text {start }}$ & $\mathrm{T}_{\exp }(s)$ & Airmass (start-end) & Solar analog (airmass) & VIS slope $\left(\mu \mathrm{m}^{-1}\right)$ & NIR1 slope $\left(\mu \mathrm{m}^{-1}\right)$ & NIR2 slope $\left(\mu \mathrm{m}^{-1}\right)$ \\
\hline A & 2011-06-24 & 01:08 & $1 \times 900$ & $1.21-1.17$ & SA110-361 (1.07) & $0.116 \pm 0.025$ & $0.021 \pm 0.040$ & $0.025 \pm 0.035$ \\
\hline B & 2011-06-24 & 02:05 & $2 \times 900$ & $1.08-1.04$ & SA110-361 (1.07) & $-0.827 \pm 0.019$ & $0.038 \pm 0.021$ & $0.076 \pm 0.026$ \\
\hline $\mathrm{C}$ & 2011-06-24 & $03: 36$ & $2 \times 900$ & $1.01-1.02$ & SA110-361 (1.07) & $-0.900 \pm 0.021$ & $-0.009 \pm 0.021$ & $0.080 \pm 0.030$ \\
\hline
\end{tabular}

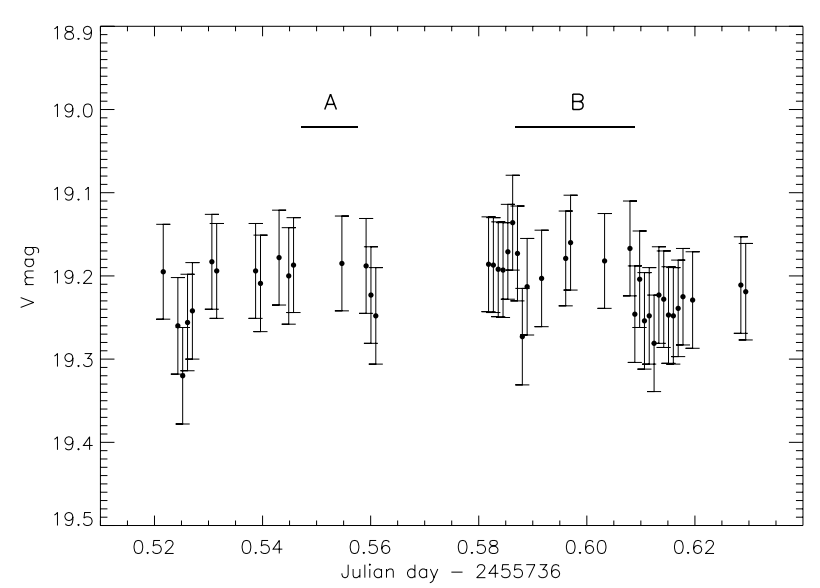

Fig. 1. Light curve of 2001 SN263 from FORS2 observations. The coverage of the obtained spectra is shown at the top. Spectrum C was obtained after the end of photometric observations.

observational conditions. To improve the physical characterization of 2001 SN263, we hence performed spectroscopic observations of this object at different rotational phases, making use of the X-Shooter spectrograph mounted at the Very Large Telescope (VLT) of the European Southern Observatory (ESO), Chile. X-Shooter spectra obtained in the UV-to-NIR range in a single shot allow properly investigating the composition of the asteroid, mapping its surface with the same observational conditions - rotational phase, aspect angle, phase angle, airmass - for the whole spectral range, and can be reliably compared with laboratory spectra of minerals and meteorites. We also performed complementary photometric observations of 2001 SN263 using the ESO-VLT FORS2 instrument. By analyzing the light curve of 2001 SN263, we aimed to determine the rotational phase and triple system geometry at the moment of the spectra acquisition.

\section{Observations and data reduction}

\subsection{FORS2}

Visible photometry was performed with the FORS2 instrument, using the standard resolution (SR) collimator and the broadband $V$ filter centered at $0.557 \mu \mathrm{m}$. We applied a $2 \times 2$ binning and an exposure time of $40 \mathrm{~s}$ for each of the images, acquired between 00:31 UT and 03:06 UT of June 24, 2011.

The images were reduced using standard procedures with the MIDAS software: subtraction of the bias from the raw data, flatfield correction, and aperture photometry to measure the instrumental magnitudes. We discarded images where the flux measurement was affected by the numerous field stars. The absolute calibration of the magnitudes was obtained by means of the observation of standard fields from the Graham (1982) and Landolt (1992) catalogs. The obtained light curve is shown in Fig. 1.

\subsection{X-Shooter}

Spectroscopy of 2001 SN263 was performed with X-Shooter on June 24, 2011, at three different times (hereafter: spectra A, B,

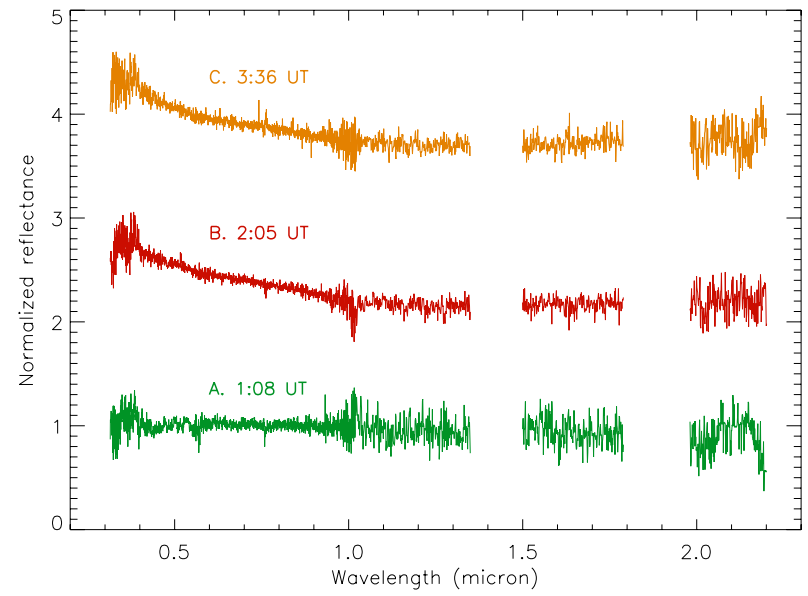

Fig. 2. X-Shooter spectra of 2001 SN263, obtained on June 24, 2011. We removed spectral regions affected by strong atmospheric absorption.

C), under a phase angle of $\sim 8.2^{\circ}$. The observational circumstances are reported in Table 1.

For the UVB and VIS arms, we used the high-gain readout mode and a $2 \times 1$ binning (readout and binning are fixed for the NIR detector), acquiring the spectra through slit widths of $1.3^{\prime \prime}$, $1.2^{\prime \prime}$, and $1.2^{\prime \prime}$ for the UVB, VIS, and NIR arms, respectively. The spectra were reduced using the X-Shooter pipeline (version 1.3.7). Performed steps were flat-fielding, wavelength calibration (using the ThAr lamp emission lines), merging of the different Echelle orders, and extraction of the spectra. Calibration files taken during daytime and a set of static files provided as part of the pipeline were used for the reduction. The reflectance of 2001 SN263 was finally obtained by dividing its spectra by that of the solar analog star SA110-361, observed at 2:48-03:00 UT of the same night. The resulting spectra are presented in Fig. 2.

\section{Data analysis and discussion}

\subsection{Light curve}

We performed a Fourier analysis of the light curve following the method developed by Harris et al. (1989). Although a number of possible solutions are found (at $1.191 \mathrm{~h}, 2.125 \mathrm{~h}, 2.943 \mathrm{~h}$, etc.), their significance is low and our data do not unambiguously agree with any periodicity, including the best solution for the rotational period given by Becker et al. (2014).

This is more probably due to the too short time-interval spanned by our observations. Unidentified mutual events, like those already reported by Betzler et al. (2008), could also have flattened the observed light curve.

\subsection{Spectra}

Although they are very noisy at wavelengths $<0.4 \mu \mathrm{m}$, the spectra seem to present a reflectance downturn starting at $\approx 0.36-0.37 \mu \mathrm{m}$. All of the three obtained spectra fall in the 
B class of the asteroid taxonomy defined by DeMeo et al. (2009). But, there is a clear variation between spectrum $\mathrm{A}$ and the remaining two spectra: the former is overall flat, while the other two present an extremely blue slope at visible wavelengths, making 2001 SN263 the object with the bluest spectrum observed so far for small bodies in the solar system (cf. Licandro et al. 2007; Clark et al. 2010; de León et al. 2012). In Table 1 we report the measured continuum slope of the spectra in three wavelength regions: $0.45-0.7 \mu \mathrm{m}$ (VIS), 1.1-1.6 $\mu \mathrm{m}$ (NIR1), and 1.6-2.2 $\mu \mathrm{m}$ (NIR2).

The airmasses at which the calibration star and 2001 SN263 were observed (cf. Table 1), raise the question whether the behavior of spectrum $\mathrm{A}$ is affected by differential atmospheric refraction (slit losses due to this effect can be intense in the UV and become negligible in the NIR, and increase with increasing airmass and displacement between the orientation of the slit and the parallactic angle). In this context, we stress that i) all of the spectra were acquired by aligning the slit with the parallactic angle; ii) X-Shooter is provided with atmospheric dispersion corrector prisms in the optical path to compensate for the atmospheric dispersion at different airmasses and minimize the losses by differential refraction; iii) some spectral variation is observed up to NIR wavelengths. Hence the observed variability is probably caused by a varying surface in the 2001 SN263 system. Unfortunately, the light curve we obtained does not help us to determine whether we observe a variation with the rotational phase of the primary body or different surface properties among the components of the triple system.

The convex shape of spectra $\mathrm{B}$ and $\mathrm{C}$ is reminiscent of that of other B-types like (24) Themis or (142) Polana. Noteworthy, the Polana family has been identified as a probable source of primitive NEAs (e.g., Campins et al. 2013). The low albedo, blue visible spectral slope, and red NIR spectral slope, make 2001 SN263 an outlier in the clustering scheme of B-types by de León et al. (2012), and CI chondrites (then coarser CMs) its most compatible meteorite analogs (e.g., Clark et al. 2011). We compared our X-Shooter spectra with mineral and meteorite spectra from the RELAB database (Pieters and Hiroi 2004). For such comparisons, care must be taken because a number of factors can affect the different spectra. For example, terrestrial weathering can contaminate the meteoritic samples, and space weathering can influence the spectra of asteroids (e.g., Clark et al. 2002); in addition, as we discuss below, particle size effects can also be strong. In Fig. 3 we show the best matches (based on chisquare minimization) we obtained for spectrum B (very consistent results are obtained for spectrum C). The only meteorite sample for which we obtain a good fit for the whole $0.3-2.2 \mu \mathrm{m}$ range is a large chip of the anomalous CI chondrite Y-82162. This meteorite, partly covered by a black fusion crust, is one of the only two CI-like thermally metamorphosed chondrites so far discovered (Tonui et al. 2014), and - as CIs in general - is very rich in phyllosilicates and magnetite (Zolensky et al. 1989). For comparison, we also show the spectrum of a sample of the CI chondrite Ivuna that has been heated to $700{ }^{\circ} \mathrm{C}$. Noteworthy, unheated samples of Ivuna and samples of the interior parts of Y-82162 do not exhibit the overall blue slope and have a reflectance downturn at longer wavelengths. This agrees with Hiroi et al. (1996), who suggested that B-type asteroids may be heated inner portions of once larger bodies and that $\mathrm{CI} / \mathrm{CM}$ meteorites may have come from the lost outer portions, which escaped extensive late-stage heating events. Among minerals in the RELAB database, a good fit is found with a sample of lampblack (a subtype of carbon black) as well as with a mixture containing 95\% montmorillonite (a phyllosilicate) and 5\% carbon

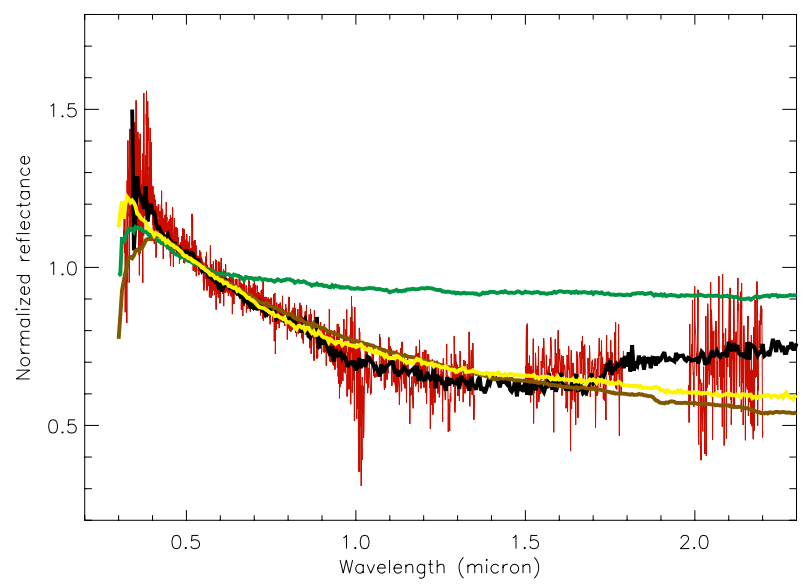

Fig. 3. Spectrum (B) of 2001 SN263, compared with those of a coated chip of unusual CI Y-82162 (in yellow, RELAB sample MB-CMP-019-CB), heated Ivuna (in green, RELAB sample MP-TXH-018-E), lampblack (in black, RELAB sample SC-EAC-024), montmorillonite mixed with carbon black (in brown, RELAB sample XM-CMP-014).

black (i.e., a material produced by the incomplete combustion of organics compounds).

All of the above depicts a consistent scenario, if we consider that organic matter, phyllosilicates and magnetite are more abundant in CIs than in other types of chondrites (e.g., Clark et al. 2011, and references therein), and that very similar spectral analogs have been found for B-type NEAs (3200) Phaethon (Licandro et al. 2007; de León et al. 2010a, 2012) and (101955) Bennu (Campins et al. 2010; Clark et al. 2011), the asteroids whose spectra (although less blue) most resemble that of 2001 SN263. The main discrepancy in this picture is the different albedo of the suggested spectral analogs (about $0.8 \%$, $4.4 \%, 9.2 \%, 11 \%$, for the lampblack, heated Ivuna, Y-82162 chip, montmorillonite and carbon black mixture, respectively) with respect to that of $2001 \mathrm{SN} 263\left(p_{V}=0.048 \pm 0.015\right)$. However, more complex mixtures may produce better fits, and the measured asteroid albedo may mainly refer to a part of the surface (or component of the triple asteroid) other than those sampled by our observations. Moreover, it is well established (e.g., Johnson \& Fanale 1973) that most carbonaceous chondrite spectra become darker with increasing grain size. Larger grains also exhibit blue-sloped spectra and a reflectance downturn starting at shorter wavelengths than fine-grained sample spectra. Such particle size effects have already been invoked to explain the differences between the visible spectra and albedo of B-type asteroid (2) Pallas and its family (de León et al. 2010b). A coarse-grained surface for 2001 SN263 could possibly explain the above mismatch and would also support the hypothesis by Delbo et al. (2011): these authors suggested a regolithdepletion mechanism during binary asteroid formation and noted that a unique system (i.e., very spaced triple components) such as 2001 SN263 could potentially be dramatically affected by this effect. Noteworthy, surface variations possibly related with binary nature have already been suggested for the NEA (175706) 1996 FG3 (Perna et al. 2013a; de León et al. 2013).

The best matches we found for spectrum A (Fig. 4) are given by the $\mathrm{CV} 3$ chondrite Allende (albedo $\sim 6.5 \%$ ) and a heated sample $\left(400{ }^{\circ} \mathrm{C}\right.$, albedo $\sim 48 \%$ ) of antigorite, another hydrated silicate. An analogy of 2001 SN263 with a CV3 chondrite (Grosnaja) was already suggested by Reddy et al. (2008) based on their NIR spectrum of the asteroid. The agreement with Allende is poor shortward of $\sim 0.43 \mu \mathrm{m}$, but our spectrum is very 


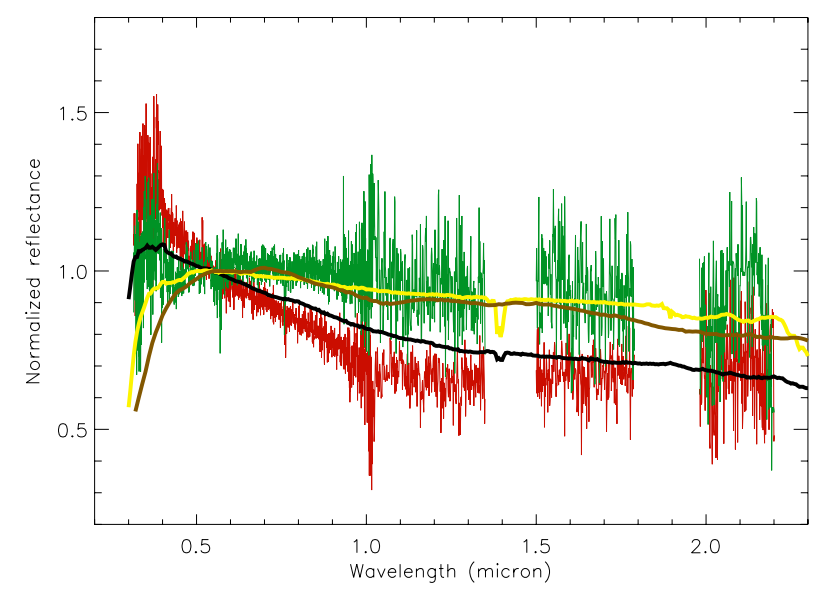

Fig. 4. Spectra of 2001 SN263 (A and B, colors as in Fig 2), compared with those of CV3 Allende (in brown, RELAB sample MT-BEC-092-A), heated antigorite (in yellow, RELAB sample AT-TXH-006), antigorite mixed with lampblack (in black, RELAB sample XT-TXH-056).

noisy in this region. The high albedo of pure antigorite is not compatible with that of 2001 SN263. But, in Fig. 4 we also show the spectrum of a mixture containing antigorite $(98.4 \mathrm{wt} \%)$ and lampblack (1.6 wt\%): this mixture has a much lower albedo of $15 \%$ and an overall shape somewhat halfway between the flat and the blue spectra of 2001 SN263.

Again, a more complex model of the surface composition may provide better results; but here our aim is to show that the mixing of a limited number of compatible, CI-like materials can produce reasonable matches with the spectra of 2001 SN263. It is also possible that the obtained data are the result of a (linear?) combination of different spectral properties on the surface of the triple system components, although we cannot determine the exact cause of such variability.

\section{Conclusions}

The Brazilian Aster space mission plans to explore (153591) 2001 SN263, one of the only two known triple near-Earth asteroids. The UV-to-NIR $(0.3-2.2 \mu \mathrm{m})$ spectra we acquired with the ESO-VLT X-Shooter instrument show that the B-type 2001 SN263 indeed represents a very intriguing target to be explored by a spacecraft.

Its ultra-blue visible spectral slope makes 2001 SN263 an extreme case among the small bodies of the solar system. From a comparison with laboratory spectra of meteorites and minerals in the RELAB database, we find that - as for some other B-types that are assumed to be samples probably coming from the heated inner portions of their parent bodies - the spectral properties of 2001 SN263 are similar to those of (naturally or artificially) heated CI carbonaceous chondrites; its surface is probably very rich in organics, magnetite, and phyllosilicates.

Remarkable characteristics of 2001 SN263 seem to be the absence of fine-grained regolith on the surface, as well as an outstanding spectral variability. These variations are probably connected with a surface variability of the primary and/or different properties among the components of the triple system. Both the large grain size and surface variability could be a direct consequence of the triple system formation (cf. Delbo et al. 2011), and the Aster mission will have the intriguing possibility of checking the scenarios predicted by models of asteroid binary formation.
The interest of visiting 2001 SN263 and study its physical properties in detail is increased by the consideration that primitive asteroids probably played a strong role in the delivery of water and complex organic molecules to the early Earth (e.g., Morbidelli et al. 2000; Izidoro et al. 2013); currently, they also pose a special hazard to our planet because the most promising techniques to deviate a small body from hazardous trajectories are much less efficient for low-density porous objects (e.g., Perna et al. 2013b).

Acknowledgements. We thank Humberto Campins, referee of this Letter, for his very helpful comments. D.P. and Z.K. acknowledge financial support from the NEOShield project, funded by the European Commission's Seventh Framework Programme (Contract No. FP7-SPACE-2011-282703). The research of Z.K. is supported by VEGA - The Slovack Agency for Science, Grant No. 2/0032/14. A.A.-C. thanks CNPq and FAPERJ for financial support through diverse grants and fellowships. O.C.W., S.M.G.W. and E.V.N. acknowledge financial support from FAPESP (proc. 2011/08171-3), CNPq and INCT Estudos do Espaço. This program was supported by the French INSU-PNP program.

\section{References}

Araujo, R. A. N., Winter, O. C., Prado, A. F. B. A., \& Sukhanov, A. 2012, MNRAS, 423, 3058

Becker, T., Howell, E., Nolan, M., et al. 2014. ACM, Helsinki, abstract B377

Benner, L. A. M., Ostro, S. J., Magri, C., et al. 2008, Icarus, 198, 294

Betzler, A. S., Novaes, A. B., \& Celedon, J. H. Q. 2008, Minor Planet Bull., 35, 182

Campins, H., Morbidelli, A., Tsiganis, K., et al. 2010, ApJ, 721, L53

Campins, H., de León, J., Morbidelli, A., et al. 2013, AJ, 146, 26

Clark, B. E., Hapke, B., Pieters, C., \& Britt, D. 2002, in Asteroids III, eds. W. F. Bottke Jr., A. Cellino, P. Paolicchi, \& R. P. Binzel (Tucson: Univ. of Arizona Press), 585

Clark, B. E., Ziffer, J., Nesvorny, D., et al. 2010, J. Geophys. Res., 115, E6005

Clark, B. E., Binzel, R. P., Howell, E. S., et al. 2011, Icarus, 216, 462

de León, J., Licandro, J., Serra-Ricart, M., Pinilla-Alonso, N., \& Campins, H. 2010a, A\&A, 517, A23

de León, J., Campins, H., Tsiganis, K., Morbidelli, A., \& Licandro, J. 2010b, A\&A, 513, A26

de León, J., Pinilla-Alonso, N., Campins, H., Licandro, J., \& Marzo, G. A. 2012, Icarus, 218, 196

de León, J., Lorenzi, V., Alí-Lagoa, V., et al. 2013, A\&A, 556, A33

Delbo, M., Walsh, K., Mueller, M., Harris, A. W., \& Howell, E. S. 2011, Icarus, 212,138

DeMeo, F. E., Binzel, R. P., Slivan, S. M., \& Bus, S. J. 2009, Icarus, 202, 160

Graham, J. A. 1982, PASP, 94, 244

Harris, A. W., Young, J. W., Bowell, E., et al. 1989, Icarus, 77, 171

Hiroi, T., Zolensky, M. E., Pieters, C. M., \& Lipschutz, M. E. 1996, Meteor. Planet. Sci., 31, 321

Izidoro, A., de Souza Torres, K., Winter, O. C., \& Haghighipour, N. 2013, ApJ, 767,54

Johnson, T. V., \& Fanale, F. P. 1973, JGR, 78, 8507

Jones, T., Lee, P., Bellerose, J. et al. 2011, LPICo, 1608, 2695

Landolt, A. U. 1992, AJ, 104, 340

Licandro, J., Campins, H., Mothé-Diniz, T., Pinilla-Alonso, N., \& de León, J. 2007, A\&A, 461, 751

Morbidelli, A., Chambers, J., Lunine, J. I., et al. 2000, Meteor. Planet. Sci., 35, 1309

Ostrowski, D. R., Lacy, C. H. S., Gietzen, K. M., \& Sears, D. W. G. 2011, Icarus, 212, 682

Perna, D., Dotto, E., Barucci, M. A., et al. 2013a, A\&A, 555, A62

Perna, D., Barucci, M. A., \& Fulchignoni, M. 2013b, A\&ARv, 21, 65

Pieters, C. M., \& Hiroi, T. 2004, LPI, 35, 1720

Reddy, V., Gaffey, M. J., Schaal, M., \& Takir, D. 2008, LPICo, 1405, 8244

Shoemaker, E. M., \& Helin, E. F. 1978, NASA CP, 2053, 245

Sukhanov, A. A., Velho, H. F. De C., Macau, E. E., \& Winter, O. C. 2010, Cosm. Res., 48, 443

Tonui, E., Zolensky, M., Hiroi, T., et al. 2014, Geochim. Cosmochim. Acta, 126, 284

Zolensky, M. E., Barrett, R. A., \& Prinz, M. 1989, Abstracts of the Lunar and Planetary Science Conf. I, 20, 1253 